\title{
Three-Dimensional X-ray Diffuse Scattering Study of Local Atomic Arrangements in $\mathrm{Ni}_{4} \mathrm{Mo}^{*}$
}

\author{
By B. Chakravarti $\uparrow$ C. J. Sparks, $₫$ E. A. Starke $\dagger$ and R. O. Williams $\ddagger$ \\ $\dagger$ Georgia Institute of Technology, Atlanta, Ga. 30332, U.S.A. \\ and $₫$ Oak Ridge National Laboratory, Oak Ridge, TN 37830, U.S.A.
}

(Received 29 April 1974)

\begin{abstract}
To construct a three-dimensional model of the local atomic arrangements for solid solutions, it is preferable to have X-ray diffuse scattering measurements in three-dimensional space. Two such measurements have been made on a single crystal of $\mathrm{Ni}_{4} \mathrm{Mo}$ as quenched from $1000^{\circ} \mathrm{C}$ and as annealed for $5 \mathrm{~min}$ at $650^{\circ} \mathrm{C}$. Following the procedure given by B. Borie \& C. J. Sparks [Acta Cryst. (1971). A 27, 198-201], the intensity was separated into that associated with chemical order and first and second-order atomic displacements. Three-dimensional Warren order coefficients and atomic-displacement coefficients were recovered. The atomic configuration was derived by computer simulation of seven of the Warren order coefficients. Visual display of the computer simulation reveals the preference of the Mo atoms to align along [100] directions of the face-centered cubic cell. As quenched, Mo atoms had a greater than average probability to form as Mo-Mo pairs when separated by the 400 interatomic vector (eighth-nearest neighbours). An even stronger rodlike morphology of the Mo-Mo pairs was observed after annealing, tending more toward the ordered form of $\mathrm{Ni}_{4} \mathrm{Mo}$. Compared with the interpretations from two-dimensional X-ray data [Spruiell, J.E. \& Stansbury, E. E. (1965). J. Phys. Chem. Solids, 26, 811-822] and electron and field ion microscopy [Ruedl, E., Delavignette, P. \& Amelincx, S. (1968). Phys. Stat. Sol. 28, 305-328; Okamoto, P. R. \& Thomas, G. (1971). Acta Met. 19, 825-841], the concept of small, perfectly ordered regions (microdomains) did not emerge from the computer simulation. Instead, the local arrangement is better described in terms of imperfect groupings with Mo atoms having a strong preference for Ni first neighbours and Mo third and eighth neighbours. The atomic displacements are small. One of the necessary criteria for the model is the reproduction of the intensity distribution and not just a comparison of the Warren order coefficients.
\end{abstract}

* Research sponsored by the United States Atomic Energy Commission. This paper has been accepted for publication in J. Phys. Chem. Solids.

J. Appl. Cryst. (1975). 8, 115

\section{A Method for the Recovery of Local Order Coefficients from Two-Dimensional Intensity Data for Binary F.c.c. Alloys}

\author{
BY J.E. EPPERSON AND P. FÜRNROHR \\ Max-Planck-Institut für Metallforschung, Institut für Werkstoffwissenschaften, Stuttgart, Germany (BRD)
}

(Received 29 May 1974; accepted 6 September 1974)

\begin{abstract}
A method is derived which permits the Cowley-Warren order parameters to be recovered from diffuse scattering measurements made in one plane in reciprocal space for systems with only short-range correlations. The procedure is demonstrated first by application to some at-temperature measurements on $\mathrm{Cu}_{3} \mathrm{Au}$ and then by considering some simulated diffraction profiles, with and without controlled errors. From the simulated scattering without error, an empirical rule is developed which allows one to decide if a given profile is amenable to this recovery method. By simulation of data with varying constant-background contributions, it is shown that such an error can be recognized and corrected.
\end{abstract}

\section{Introduction}

It is well known that the physical properties of an alloy may be influenced, to varying degrees, by the existing state of local atomic order. Cowley (1950) has shown that the statistical arrangement of the atoms on the average lattice sites can be determined from a Fourier analysis of the local-order-modulated Laue monotonic diffuse scattering. In most real systems, the diffusely scattered profile is more complicated because of the 